OPEN ACCESS

Edited by:

Leonid Goubergrits,

Charité-Universitätsmedizin

Berlin, Germany

Reviewed by:

Filippo Cademartiri,

IRCCS SDN, Italy

Christos Rammos,

University of

Duisburg-Essen, Germany

*Correspondence:

Markus U. Wagenhäuser markus.wagenhaeuser@

med.uni-duesseldorf.de

tThese authors have contributed equally to this work

*These authors have contributed equally to this work and share senior authorship

Specialty section: This article was submitted to

Cardiovascular Imaging

a section of the journal

Frontiers in Cardiovascular Medicine

Received: 16 May 2021

Accepted: 02 July 2021

Published: 13 August 2021

Citation:

Logghe G, Trachet B, Segers P, De

Backer J, Mulorz J, Dueppers $P$

Vermassen F, Schelzig H, Van

Herzeele I and Wagenhäuser MU (2021) Outflow Through Aortic Side

Branches Drives False Lumen Patency

in Type B Aortic Dissection.

Front. Cardiovasc. Med. 8:710603.

doi: 10.3389/fcvm.2021.710603

\section{Outflow Through Aortic Side Branches Drives False Lumen Patency in Type B Aortic Dissection}

\author{
Gerlinde Logghe ${ }^{1 \dagger}$, Bram Trachet ${ }^{1 \dagger}$, Patrick Segers ${ }^{1}$, Julie De Backer ${ }^{2}$, Joscha Mulorz ${ }^{3}$, \\ Philip Dueppers ${ }^{3,4}$, Frank Vermassen ${ }^{5}$, Hubert Schelzig ${ }^{3}$, Isabelle Van Herzeele ${ }^{5 \neq}$ and \\ Markus U. Wagenhäuser ${ }^{3 * \neq}$ \\ ${ }^{1}$ Institute for Biomedical Engineering and Technology, Ghent University, Ghent, Belgium, ${ }^{2}$ Department of Cardiology and \\ Center for Medical Genetics, Ghent University Hospital, Ghent, Belgium, ${ }^{3}$ Department of Vascular- and Endovascular \\ Surgery, Medical Faculty and University Hospital Düsseldorf, Heinrich-Heine-University Düsseldorf, Düsseldorf, Germany, \\ ${ }^{4}$ Department of Vascular Surgery, University Hospital Zurich, University of Zurich, Zurich, Switzerland, ${ }^{5}$ Department of \\ Thoracic and Vascular Surgery, Ghent University Hospital, Ghent, Belgium
}

Objective: Thoracic endovascular aortic repair (TEVAR) for type B aortic dissection (TBAD) aims to induce false lumen (FL) thrombosis by sealing intimal tears between the true $(T L)$ and the $F L$, and blocking the inflow into the FL. Incomplete thrombosis of the $\mathrm{FL}$ is correlated with poor clinical outcome. We hypothesize that the number of major and minor branches arising from the FL affects FL patency and may negatively influence TEVAR induced FL thrombosis.

Methods: Computed tomography (CT)-scans from 89 patients diagnosed with TBAD [best medical treatment (BMT) $n=52$, TEVAR $n=37$ ] from two high-volume vascular surgery centers were analyzed retrospectively. Analysis included evaluation of the FL patency status, the number, location and size of intimal tears, and the presence of minor and major side branches originating from the FL. Multiple regression analysis was conducted to evaluate obtained parameters as predictors for FL thrombosis status.

Results: In univariate analysis, the strongest correlation for FL patency was found for the number of major $(R=0.79)$ and minor $(R=0.86)$ side branches originating from the FL. When applying a multiple linear regression model, the number of major (normalized beta $0.37 ; P<0.001$ ) and minor (normalized beta $0.41 ; P<0.01$ ) side branches arising from the FL were valid predictors for the axial length of the patent and non-patent FL, and additionally determined the length of the patent FL at 12-month follow-up in patients that underwent TEVAR.

Conclusions: Our data suggest that the number of minor side branches that originate from the FL in TBAD is an important determinant of FL patency, to a greater degree than previously assumed.

Keywords: aortic dissection, minor branches, outflow, patency, TEVAR, false lumen, true lumen 


\section{INTRODUCTION}

Type B aortic dissection (TBAD) is defined as a tear in the intimal layer of the descending thoracic aorta (DTA) distal to the left subclavian artery (LSA), with consequent blood flow within the medial layer of the aorta (1). Mortality rates vary significantly depending on the acute or chronic nature of the pathology, occurrence of complications and treatment chosen (1-5). Overall pre- and post-discharge mortality and morbidity are high for all types of TBAD, and 5-year patient survival rates range from 50 to $70 \%(1,5,6)$. Well over $60 \%$ of deaths associated with acute TBAD result from aortic rupture, mostly located at the false lumen $(\mathrm{FL})(1,4,6,7)$.

Treatment of TBAD focuses on restoring or maintaining vital organ function and peripheral perfusion while preventing aortic rupture. Uncomplicated TBAD is primarily treated with antihypertensive drugs to prevent progression and aneurysm formation. However, several studies demonstrated an early benefit of thoracic endovascular aortic repair (TEVAR) when compared to antihypertensive therapy alone, although long-term results are pending thorough validation (7-11).

In patients with TBAD complicated by renal, visceral or limb ischemia, persistent pain, refractory hypertension, and rapid or chronic expansion of the aortic diameter open surgical repair (OSR) or TEVAR are often inevitable. The latter aims to seal the primary entry tear and thus reduces blood flow into the FL. By promoting FL thrombosis, TEVAR may also prevent distal extension of the dissection (1). Superior patient outcomes have been reported following TEVAR when compared with OSR $(1,12-14)$. Despite these benefits, procedurerelated complications such as stroke and progression of TBAD may occur $(5,7,15-18)$.

It is known that entry tear sizes $>10 \mathrm{~mm}$, initial FL diameter and incomplete, partial thrombosis of the FL predict the long-term mortality risk in patients with $\operatorname{TBAD}(15,17)$. Importantly, partial FL thrombosis was shown to be a significant independent predictor of post-discharge mortality $(16,18)$. Yet, studies exploring predictive factors for partial FL thrombosis are rather scarce. Maximum FL-diameter, (re-) entry tears, blindsac subtype and large visceral branches arising from the FL have been suggested to influence partial FL thrombosis $(7,19,20)$. These parameters relate to the in- and outflow into and from the FL. However, outflow from the FL does not occur only through visceral branches or re-entry tears but may also occur through smaller communicative branches such as intercostal, lumbar and bronchial arteries which may also originate from the $\operatorname{FL}(21,22)$.

In this retrospective exploratory study, we document and quantify the number of major and minor side branches arising from the FL in TBAD and evaluate their role in FL thrombosis. The study is based on the consideration that stasis of blood is a necessary condition to achieve FL thrombosis (23). When an inflow tract into the FL is present [e.g., one or several intimal tears between the true lumen (TL) and the FL], the presence of side branches arising from the FL results in hemodynamic conditions that impede complete stasis of the blood. In this context, previous observations from our group in Angiotensin-II-infused mice, a popular mouse model for aortic dissection (24-26), suggested an important role of both minor and major side branches in FL thrombosis and disease progression $(26,27)$.

The aim of the present study is to test the hypothesis that outflow from the FL through major and minor side branches affects FL patency by providing a continuous hemodynamic connection between the TL and FL.

\section{METHODS \\ Data Collection}

Medical records and vascular imaging data of patients suffering from TBAD who were treated in the Department of Vascular and Endovascular Surgery at the University Hospital Düsseldorf (UKD) and in the Department of Thoracic and Vascular Surgery at Ghent University Hospital (UZG) were screened retrospectively following approval from the local ethics committess (study ID: UKD: 2017064325; UZG: EC/2017/1635). Patients were excluded from the study if they (i) did not agree to participate, (ii) suffered from known genetic disorders, (iii) had a (history of) type A aortic dissection, (iv) were treated with OSR or (v) had poorly contrasted CT angiograms.

Baseline was defined by the first available Computed tomography (CT)-scan after admission. Demographic data (sex and age) and risk factors (pre-existing arterial hypertension, active smoking, diabetic status, statins on admission, family history of aortic dissection, and renal insufficiency) were obtained for all patients. In patients undergoing TEVAR, indications for intervention, stent graft information (number of stent grafts, coverage length) and complications during or following surgery (spinal cord ischemia, transient ischemic attack (TIA), stroke or death) were evaluated.

\section{Image Analysis}

CT images from patients from the UZG and UKD databases were acquired using state-of-the-art clinical scanners from different manufacturers (Siemens, Erlangen, Germany; Toshiba, Tokyo, Japan; Phillips Healthcare, Hamburg, Germany; Canon Medical, Otawara, Japan). Slice thickness varied from 1 to $5 \mathrm{~mm}$ for the UZG patients, and from 0.75 to $5 \mathrm{~mm}$ for the UKD patients with $5 \mathrm{~mm}$ slice thickness CT-scans in 3 patients across both institutions. Image analysis was performed using Mimics 19 (Materialize, Leuven, Belgium) and similarly applied to all datasets. Initially, the aorta was cropped from the first slice distal to the LSA down to the last axial slice above the aortic bifurcation. Within this region of interest (ROI), the aortic domain was subdivided into three different zones: TL, patent FL and nonpatent FL. Figure 1 shows three representative and segmented aortae with each of the three aforementioned zones indicated by a different color. The distinction between patent and non-patent FL was based on differences in mean gray scale values. We then quantified the number of major side branches (celiac, superior and inferior mesenteric, left and right renal arteries) and minor side branches (intercostal, lumbar, gonadal arteries, middle sacral artery) arising from the TL, the patent and non-patent FL. Each additional branch resulted in an addition of 1.0 to the cumulative 

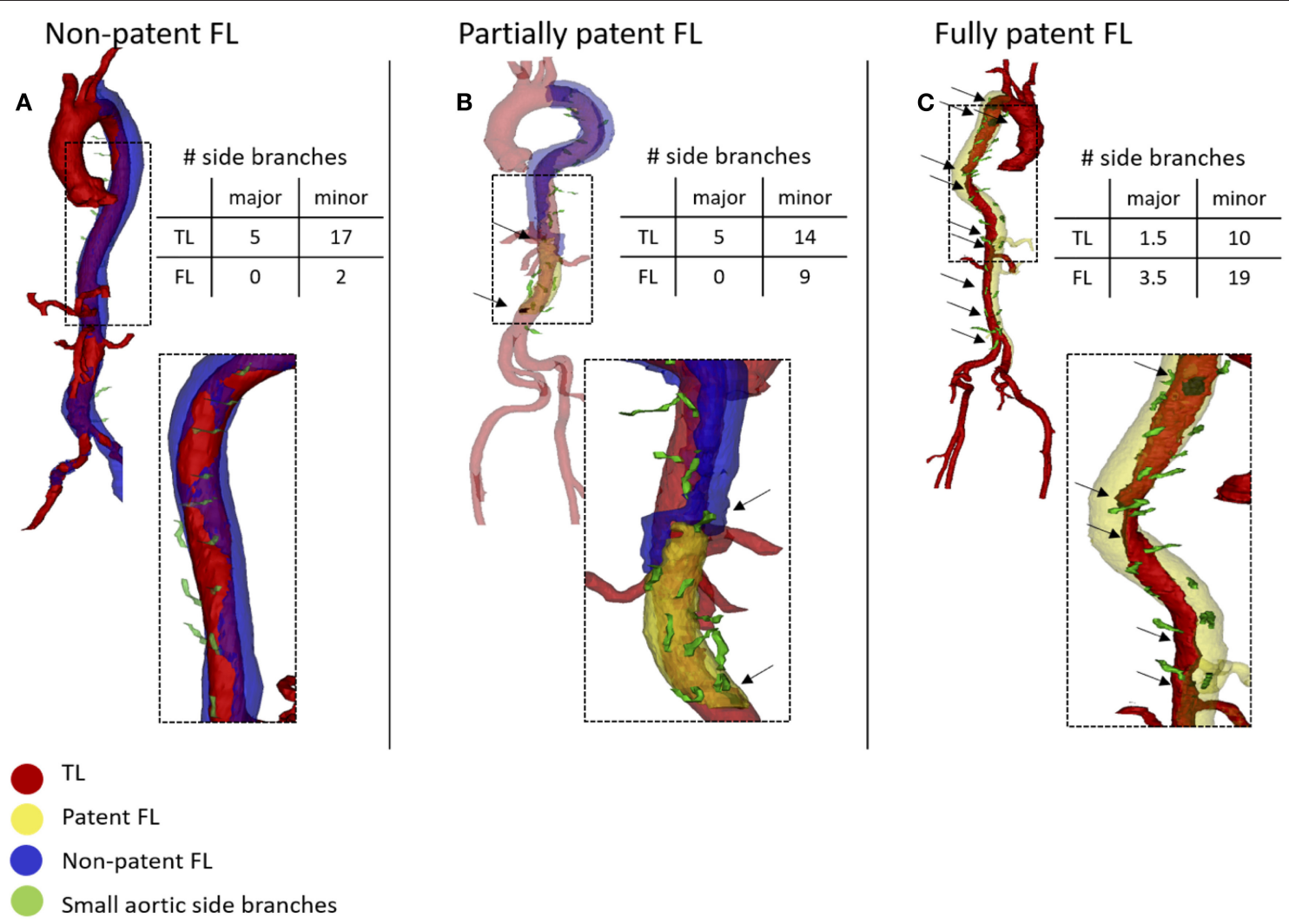

FIGURE 1 | 3D segmentation of three representative baseline Computed-tomography (CT)-scans. Black arrows indicate intimal tears. The false lumen (FL) is non-patent along its entire length (A). Non-patent FL in the proximal segment, partially patent FL at the level of major visceral branches, fully patent FL in the distal segment (B). Fully patent FL along its entire length (C). Minor branches originate from the FL in fully patent segments of the FL, while they originate from the TL in non-patent segments of the $F L(\mathbf{A})$. In partially patent $F L(\mathbf{B})$ minor branches originate from the $F L$ in the patent segment and from the $T L$ in the non-patent segment.

score. For branches that originate from both the TL and the FL, a score value of 0.5 was added.

Each axial CT slice within the ROI was subsequently assigned one of four patency labels: no FL, fully patent FL, non-patent FL or partially patent FL (when a segment of the FL was patent and another segment was non-patent) (Figure 1). The axial length of the TL, patent FL, and non-patent FL was calculated as the number of axial slices that bore the corresponding patency label, multiplied with the interaxial slice distance, which was constant for each individual scan. For the calculation of axial lengths, the patent FL was defined to include all slices with either a fully patent or a partially patent FL. The axial distance of the non-patent FL was hence based on axial CT slices in which the entire crosssection of the FL was non-patent. The relative FL cross-sectional area was quantified as the ratio of the local 2D FL lumen area surface over the complete lumen area $(\mathrm{TL}+\mathrm{FL})$.

In a next step, we quantified the number and the volume of intimal tears within the ROI. An intimal tear was defined as a disruption of the intimal flap separating the TL and FL. Intimal tears were counted if a disruption was visible on at least two of three views (axial, sagittal and/or coronal view). A disruption was attributed a score value of 0.5 , if it was only visible on one of the three views, or not clearly visible on several views. The volume of an intimal tear was defined as the volume of the tissue missing from the aortic wall at the location of the intimal tear. In order to quantify the volume of the intimal tear, the thickness of the missing tissue was assumed in correspondence to the surrounding aortic wall. The volume of intimal tears was semi-automatically quantified in 3D using Mimics. The axial distance between the LSA and the most proximal slice of the most proximal intimal tear was estimated based on the number of axial slices between both landmarks.

\section{Analysis of Baseline Data}

Rstudio (Version 1.3.959, ( ) 2009-2020 RStudio, Public Benefit Corporation) was used for statistical analyses. Mann-WhitneyWilcoxon Test was used to evaluate differences between the two institutions for the quantified parameters (ns: $p>0.05$, $\left.{ }^{* *} p \leq 0.01\right)$. Spearman's rank correlation coefficient was used to examine the correlation between the axial length of the patent segment of the FL and other parameters. Kruskal-Wallis test was used to compare the axial length of different patency zones (patent FL, non-patent FL, no FL) between the different institutions (UZG vs. UKD). Multiple regression analysis was performed for the axial length of the patent portion of the FL as dependent variable and the number and size of intimal tears, 
the distance from the LSA to the most proximal intimal tear, the relative FL area, the number of major side branches and the number of minor side branches arising from the FL, and the number of minor side branches originating from the TL as independent variables. A similar model was set up with the axial length of the non-patent portion of the FL as dependent variable. Results are presented as forest-plots of standardized beta values (dividing by two standard deviations). $P$-values $\leq 0.05$ were considered statistically significant.

\section{Analysis of Follow-Up Data}

Similar to the baseline data, the axial length of the fully patent, partially patent and non-patent FL segments of the aorta were determined, as well as of the non-dissected aorta between the LSA and the aortic bifurcation. We assessed the correlation between the axial length of the patent FL and its assumed determinants in univariate analysis and multiple linear regression models. We also compared the axial length of the patency status in the FL and the non-dissected lumen between the two treatment groups (BMT, TEVAR). For this comparative analysis, lengths were normalized to the total length of the aortic segment from the LSA to the aortic bifurcation.

\section{RESULTS}

\section{Baseline Data}

A total of 89 patients was included in this study. Given the retrospective study design, follow-up intervals varied. Followup CT-scans of patients receiving best medical treatment (BMT) were binned into five different time points: baseline $(n=$ 89), 2-4 months $(n=41), 10-14$ months $(n=16), 20-$ 28 months $(n=15), 40-56$ months $(n=4)$ after baseline (Supplementary Figure 1, upper panel). Of these, 37 patients underwent TEVAR, with a mean period from baseline to TEVAR of $126 \pm 191$ days. The last CT-scan prior to intervention was considered as TEVAR baseline. Subsequent follow-up scans were binned relative to TEVAR baseline, i.e., $2-4$ months $(n=17), 10-$ 14 months ( $n=21), 20-28$ months $(n=17)$, and 40-56 months ( $n=10$ ) following surgery (Supplementary Figure 1, lower panel). Of note, there was no spinal cord ischemia following TEVAR. Baseline demographics and risk factors are displayed in Table 1. Mean follow-up for BMT and TEVAR cohorts was 320 \pm 486 days and $993 \pm 772$ days, respectively.

\section{Functional Parameters}

The distribution of the quantified parameters for both hospitals is provided in Supplementary Figure 2. There were no significant differences between the two patient collectives, except for the average number of intimal tears/patient $(3.6 \pm 3.24$ tears/patient for UKD; $1.65 \pm 1.75$ tears/patent for UZG, Mann-WhitneyWilcoxon, $p \leq 0.01$ ).

The number of major $(R=0.79)$ and minor $(R=0.86)$ side branches arising from the FL showed the highest correlation with the axial length of the patent FL in univariate analysis (Figure 2). Other quantified parameters, such as the number $(R=0.63)$ and size $(R=0.63)$ of intimal tears, the distance from the most proximal intimal tear to the LSA $(R=-0.29)$, and the relative FL
TABLE 1 | Age, sex and risk factors on admission are displayed for all patients at baseline.

Patients at baseline $(n=89)$

\begin{tabular}{|c|c|}
\hline \multicolumn{2}{|l|}{ Age in years } \\
\hline & $65.2(13.6)$ \\
\hline \multicolumn{2}{|l|}{ Sex } \\
\hline Male/female & $72 / 17(80.9 \% / 19$ \\
\hline \multicolumn{2}{|l|}{ Risk Factors on admission* } \\
\hline Pre-existing arterial hypertension & $78(87.6 \%)$ \\
\hline Active smoking & 35 (39.3\%) \\
\hline Diabetic & $12(13.5 \%)$ \\
\hline Statin treatment & $23(25.8 \%)$ \\
\hline Family history of aortic dissection & $10(11.2 \%)$ \\
\hline Renal insufficiency & $12(13.5 \%)$ \\
\hline \multicolumn{2}{|c|}{ Patients undergoing TEVAR $(n=37)$} \\
\hline \multicolumn{2}{|l|}{ Indications for intervention } \\
\hline Refractory pain/uncontrolled hypertension & $8(21.6 \%)$ \\
\hline Malperfusion & $8(21.6 \%)$ \\
\hline Rapid enlargement of the aorta & $11(29.7 \%)$ \\
\hline Rupture & $0(0 \%)$ \\
\hline Change of disease class/type & 7 (18.9\%) \\
\hline Other/unknown e.g., surgeon's preference,... & $7(18.9 \%)$ \\
\hline \multicolumn{2}{|l|}{ Number of stent grafts } \\
\hline & $1.14(0.419)$ \\
\hline \multicolumn{2}{|l|}{ Lenght of aortic coverage (mm) } \\
\hline & $150(53.6)$ \\
\hline \multicolumn{2}{|l|}{ Complications following surgery } \\
\hline Spinal cord ischemia & $0(0 \%)$ \\
\hline TIA/stroke & $0(0 \%)$ \\
\hline Death & $2(5.4 \%)$ \\
\hline
\end{tabular}

For patients that received TEVAR treatment, indications for intervention, number of implanted stent grafts, length of aortic coverage and complications following surgery are displayed. Results are presented as absolute frequency $n$ (\%) or mean with (standard deviation).

${ }^{\star}$ Risk factors as reported in the medical records of the patients. TEVAR, Thoracic endovascular aortic repair; TIA, transient ischemic attack.

area $(R=0.68)$ correlated less strongly with FL patency, although all correlation coefficients reached statistical significance.

\section{Determinants of False Lumen Patency}

The number of minor (standardized beta $0.41, P<0.01$ ) and major side branches (standardized beta $0.37, P<0.001$ ) originating from the FL emanated as the strongest determinants of the regression model to predict the axial length of the patent portion of the FL in multiple linear regression analysis (Figure 3). The relative FL area had a (minor) positive effect (normalized beta $0.25, p \leq 0.01$ ) in the patent FL regression model. When predicting the axial length of the non-patent portion of the FL in the regression model, the distance from the first intimal tear to the LSA was the only parameter with a (minor) positive effect (normalized beta $0.23, p \leq 0.05$ ). The number of major branches arising from the FL, as well as the number of minor branches arising from the FL, had minor negative effects in this model (normalized beta $-0.23, p \leq 0.05$ and normalized beta -0.36 , 


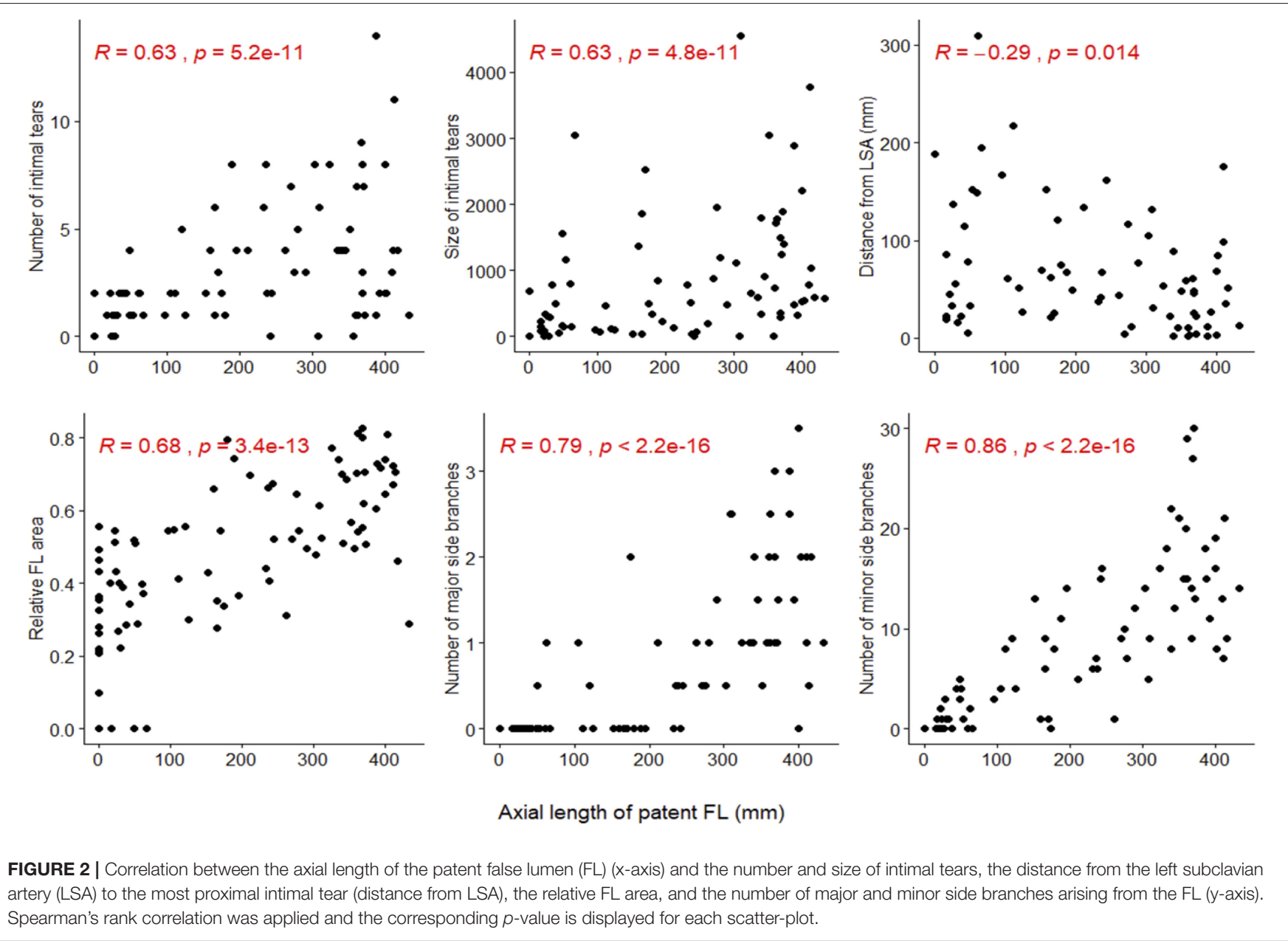

$p>0.05$, respectively). In both, the patent and non-patent FL regression model, the number and size of the intimal tears and the number of minor branches originating from the TL only marginally contribute to the prediction (Figure 3).

\section{Analysis of Follow-Up Data}

The portion (= "relative axial length") of the fully patent FL was lower for patients undergoing TEVAR when compared to patients receiving BMT (Figure 4) at all follow-up time points $(p<0.05)$. However, the relative length of the partially patent FL remained unchanged following TEVAR treatment $(p>0.05$ in all TEVAR follow-up groups). Also, no significant difference in relative length of the partially patent FL was found between the BMT vs. TEVAR group at all follow-up time points $(p>0.05)$.

Most data for TEVAR were available at 12 months followup $(n=21)$. There was a significant correlation between the patent FL length and the number of minor side branches arising from the FL in univariate analysis (Figure 5A) and multivariate linear regression analysis (Figure 5B; normalized beta $0.83, p<0.05)$. Data from other time points are provided in Supplementary Figure 3. In univariate analysis, a positive and significant relation was found between the patent FL length and the number of minor FL side branches at all time points.

\section{DISCUSSION}

At the onset of this study, we hypothesized that in patients with TBAD, outflow through major and minor branches arising from the FL maintains blood flow through the FL and thus prevents FL thrombosis. The data gathered in this retrospective study strongly supports this hypothesis. Both univariate and multiple regression analysis identified the number of branches originating from the FL as the most important predictor for lumen patency. Despite the relatively small number of TEVAR cases, follow-up data further suggest that FL side branches remain a major determinant of FL patency in patients following thoracic stent graft placement. Based on these results we advocate that FL thrombosis is unlikely to be achieved in the presence of (minor) side branches arising from a perfused FL (Figure 6).

It is important to emphasize that our investigation did not study nor question the importance of intimal tears for FL 


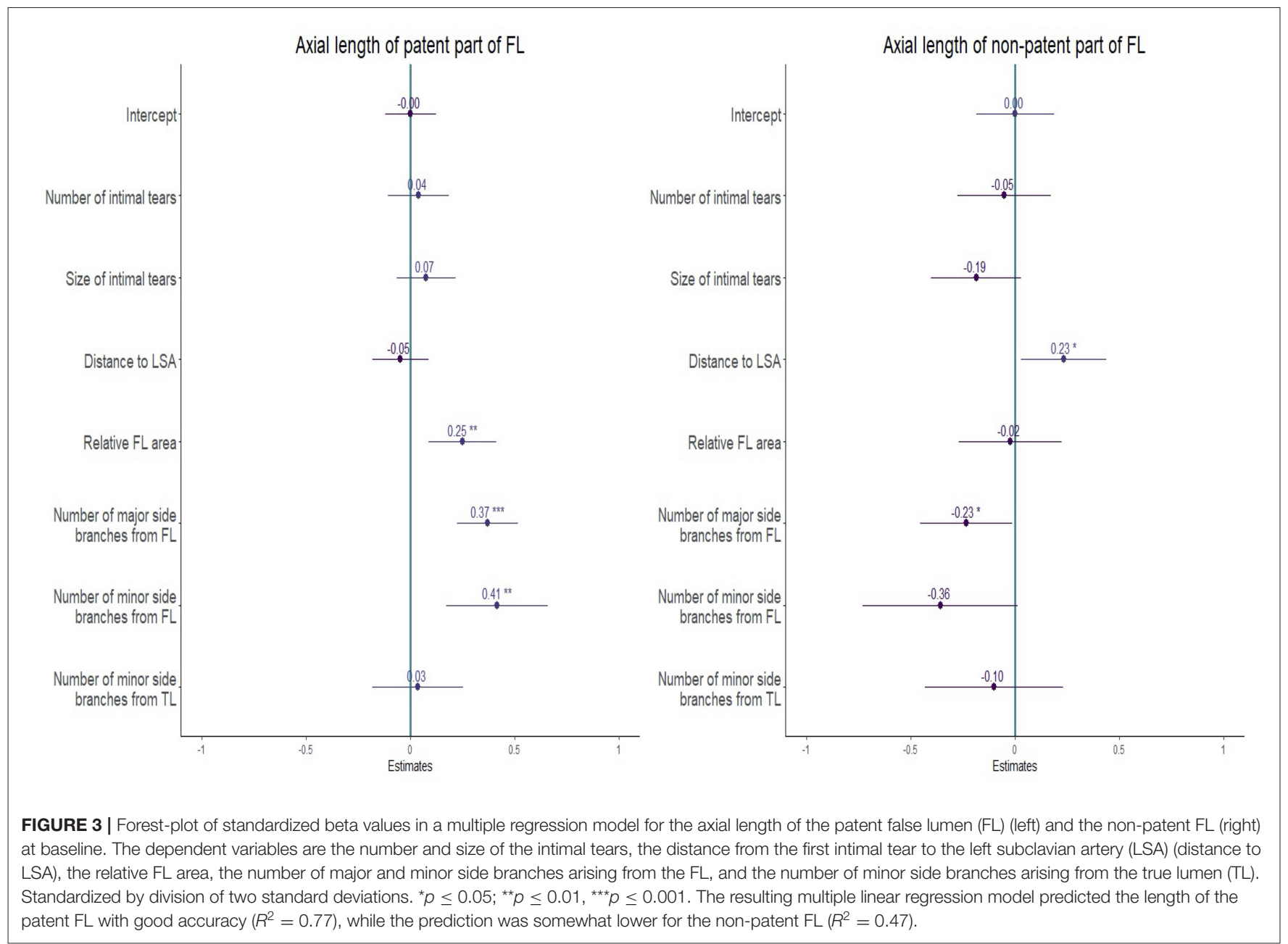

patency and patient outcomes in TBAD (17). Indeed, for an outflow to occur from the FL, inflow needs to be provided by at least one intimal tear connecting the TL and FL and our data confirm the significance of tear size and location for FL patency. However, these inflow parameters appear less significant when compared to outflow parameters such as the number of branches arising from the FL when applying multiple linear regression models.

Since minor FL branches might sustain the circulation of blood within (parts of) the FL, we further hypothesized that TEVAR treatment is less likely to induce FL thrombosis in case (i) side branches originate from the FL and (ii) blood flow into the FL is still present following TEVAR. Successful treatment by inducing FL thrombosis may therefore depend on the number of branches arising from the FL. This hypothesis seems in line with patient outcomes following TEVAR, which reported complete FL thrombosis in up to $40 \%$ of all cases while persistent distal perfusion may lead to late FL expansion in up to $30 \%$ of all cases during follow-up $(28,29)$.

Our data support the aforementioned hypothesis. TEVAR did not reduce the axial length of partial thrombosis (Figure 4). The proposed underlying mechanism is illustrated in Figure 5 (right panel) on 3D segmented aortae for two exemplary cases. Since
TEVAR may only block FL inflow at the proximal intimal entry tear, outflow through side branches arising from the FL is present if tears distal to the implanted stent graft (so-called re-entry tears) are not blocked. This leads to retrograde flow into the FL with inflow from distal tears and outflow into small side branches. In these cases, blocking the inflow from the primary entry tear by TEVAR does not result in a fully thrombosed FL but predisposes for a partially thrombosed FL with residual flow into the FL from distal tears and outflow into minor side branches. This concept is also supported by our, albeit slightly underpowered, follow-up study. Despite the low number of TEVAR follow-up cases, the follow-up data at 12 months demonstrated a significant correlation between the extent of patent FL and the number of side branches arising from the FL. The number of side branches originating from the FL was the only variable reaching statistical significance in multiple regression analysis in TEVAR patients at 12 months follow-up (Figure 5). It is important to emphasize that these observations do not question the efficacy of TEVAR treatment in general. In fact, our data support the success of TEVAR in promoting FL thrombosis. Patients treated with TEVAR had increased relative axial length of the non-patent FL when compared to those receiving BMT, which was true for all patients at all follow-up time points. 


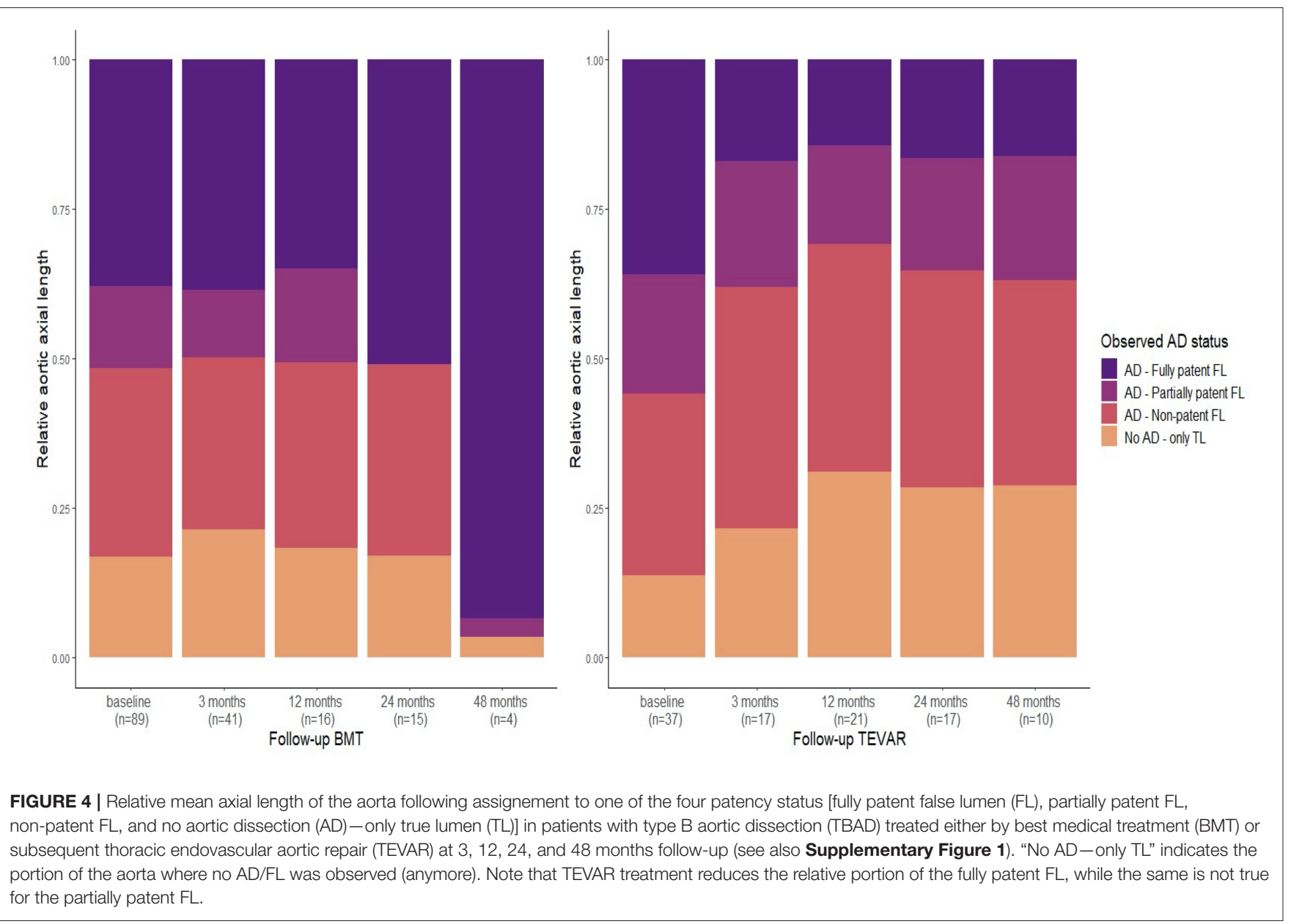

Over time, several techniques have been introduced to enhance FL thrombosis and aortic remodeling following TEVAR. Extended aortic covering utilizing longer stent grafts were found to bear a higher risk of spinal cord ischemia while seeking to occlude multiple tears between the TL and FL aiming to reduce pressure and flow transmission into the FL $(30,31)$. The socalled Knickerbocker Technique is based on the dilation of the central stent graft segment in the TL to rupture the dissection membrane aiming to punctually compress the FL (32). Other authors used FL lumen access for plug (so-called candy-plug), coil or glue deployment to block retrograde inflow and promote FL thrombosis $(33,34)$. While all these approaches aim to reduce inflow into the FL, our data advocate that controlling the outflow might be equally important. This is also supported by findings of the ADSORB trial, which noted that the number of vessels originating from the FL may predict FL growth in patients with TBAD (35). Taking this into consideration, specifically targeting side branches that originate from the FL aiming to block the outflow from the FL seems beneficial and feasible by e.g., selective embolization, since such procedures have already been described and demonstrated an effective induction of FL thrombosis in TBAD while avoiding device deployment into the FL (36). Such device deployment into the FL might hold several risks such as narrowing of the TL, damaging the dissection membrane, device migration and breakage or endoleakage. In contrast, a more specific approach aiming to occlude originating FL branches (e.g., via embolization) would minimize the amount of implanted material and device deployment-associated risks (37).

While the association between (partial) FL patency and survival rates in TBAD has been studied extensively $(28,38-$ 41 ), little is known about the underlying and causal mechanisms. It has been hypothesized that pulsatile inflow through intimal tears into a lumen with impaired outflow (when there are no re-entry tears) increases blood pressure in such FL, which in turn subsequently leads to a higher risk of lumen expansion, dissection progression and rupture $(13,16,40,41)$. However, the direct effect of inflow on FL pressure is expected to be limited while maximally arising to the dynamic pressure, which would equal $<5 \mathrm{mmHg}$ when assuming a peak aortic velocity of $\sim 1 \mathrm{~m} / \mathrm{s}$ (estimated from the simplified Bernoulli equation with dynamic pressure, in $\mathrm{mmHg}$, approximating $4 \mathrm{v}^{2}$ with $\mathrm{v}$ the velocity in $\mathrm{m} / \mathrm{s}$ ). Moreover, this hypothesis does not consider that branches arising from the FL provide an alternative outflow, thus preventing pressure build-up within the FL. From a hemodynamic perspective, the alternative hypothesis we propose here provides an elegant solution for these problems. 


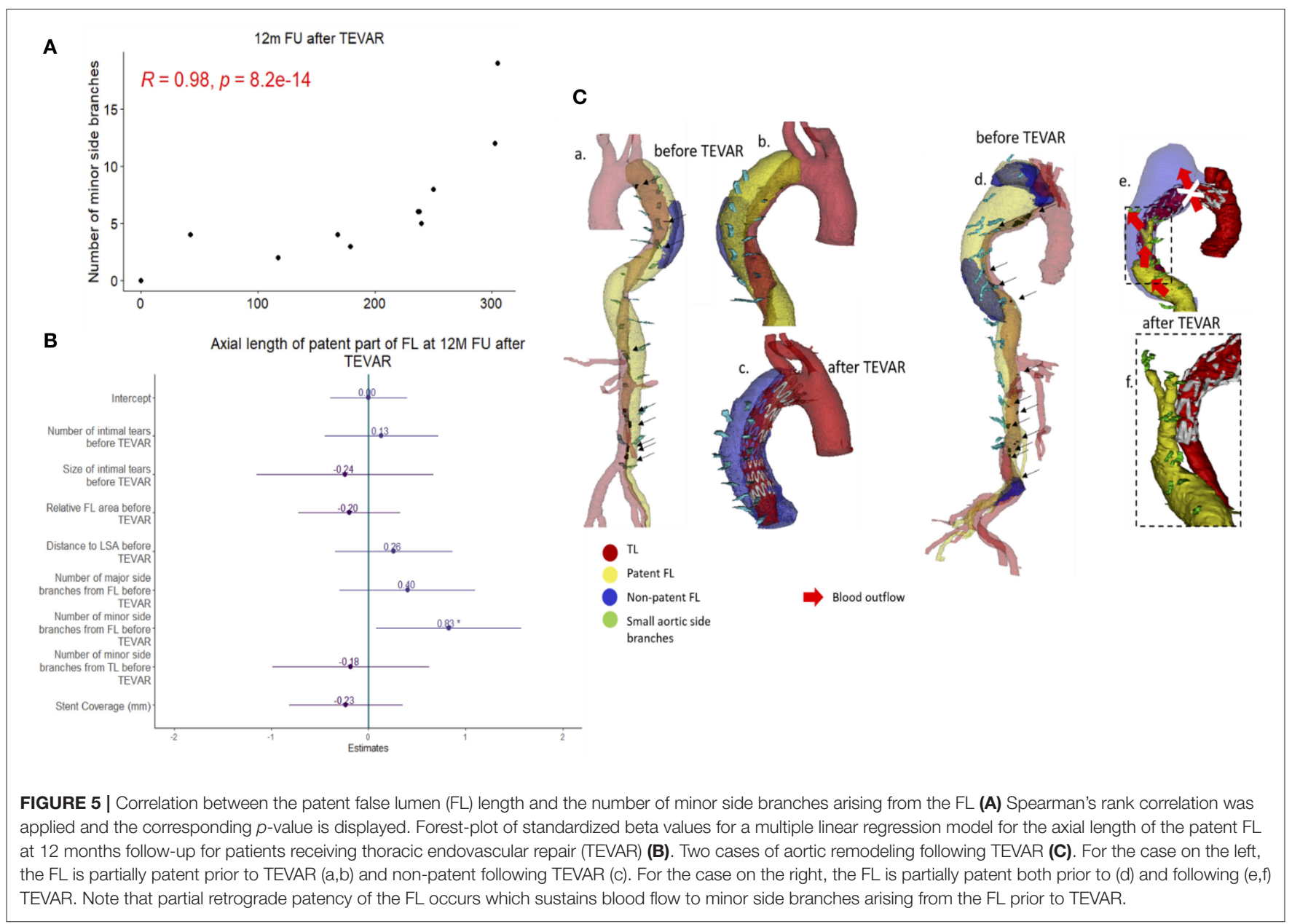

Our study has several limitations. First, only two centers retrospectively collected patient data, thus limiting cohort sizes. Next, high-resolution CT images are indispensable to properly identify minor side branches and intimal tears. Intimal tears can sometimes be mistaken for a flapping aorta, while the smallest side branches sometimes take up only few pixels on a single cross-section. All quantifications were performed directly on the images by two well-trained observers (BT and GL). Nevertheless, variations due to interpretation errors cannot be excluded, also given that there was a difference in mean slice thickness between UZG $(2.26 \pm 0.83 \mathrm{~mm})$ and UKD $(1.4 \pm 1.08 \mathrm{~mm})$. The latter may explain why UZG patients had significantly fewer intimal tears when compared with UKD patients, although there was no significant difference in the total number of detected minor side branches. Also, minor side branches arising from a thrombosed FL receive proportionally less blood flow and are thus more difficult to detect on CT images compared to minor side branches that arise from a patent FL. This may have affected the results of our study. However, we did not observe significant differences (Wilcoxon test $p>0.05$ ) in the total number of minor side branches between patients with a fully thrombosed $(22.5 \pm 4.22)$ and patients with a fully patent FL $(24.1 \pm 3.98)$. Since FL patency was determined based on differences in gray scale values, the timing of the CT-scan following contrast-bolus injection could have also affected the assessed FL patency. Finally, we approximated the axial length of the patent and the non-patent FL by multiplying the number of axial CT slices on which either was visible with the distance between two slices. In this regard, using 3D volumes instead of axial lengths, which is more labor intensive, could increase accuracy, since it would allow us to take the shape of the aorta also into account.

Potential clinical implications of the findings have not been in the scope of this exploratory study; however, generating a clinical score based on the number of arising minor branches from the FL (TL) and locations/sizes of intimal tears via smart computer algorithms to provide an estimation on whether the aorta is at risk for expansion or even whether TEVAR may be beneficial in terms of FL thrombosis induction seems generally feasible at long-term. Although potentially valuable, a cut-off value for such score needs to be defined and validated first and would further require correlation with clinical and morphological outcomes to prove its effectiveness in a preferably prospective setting. Also, potential alternative approaches, such as the specific occlusion of originating FL side branches via 


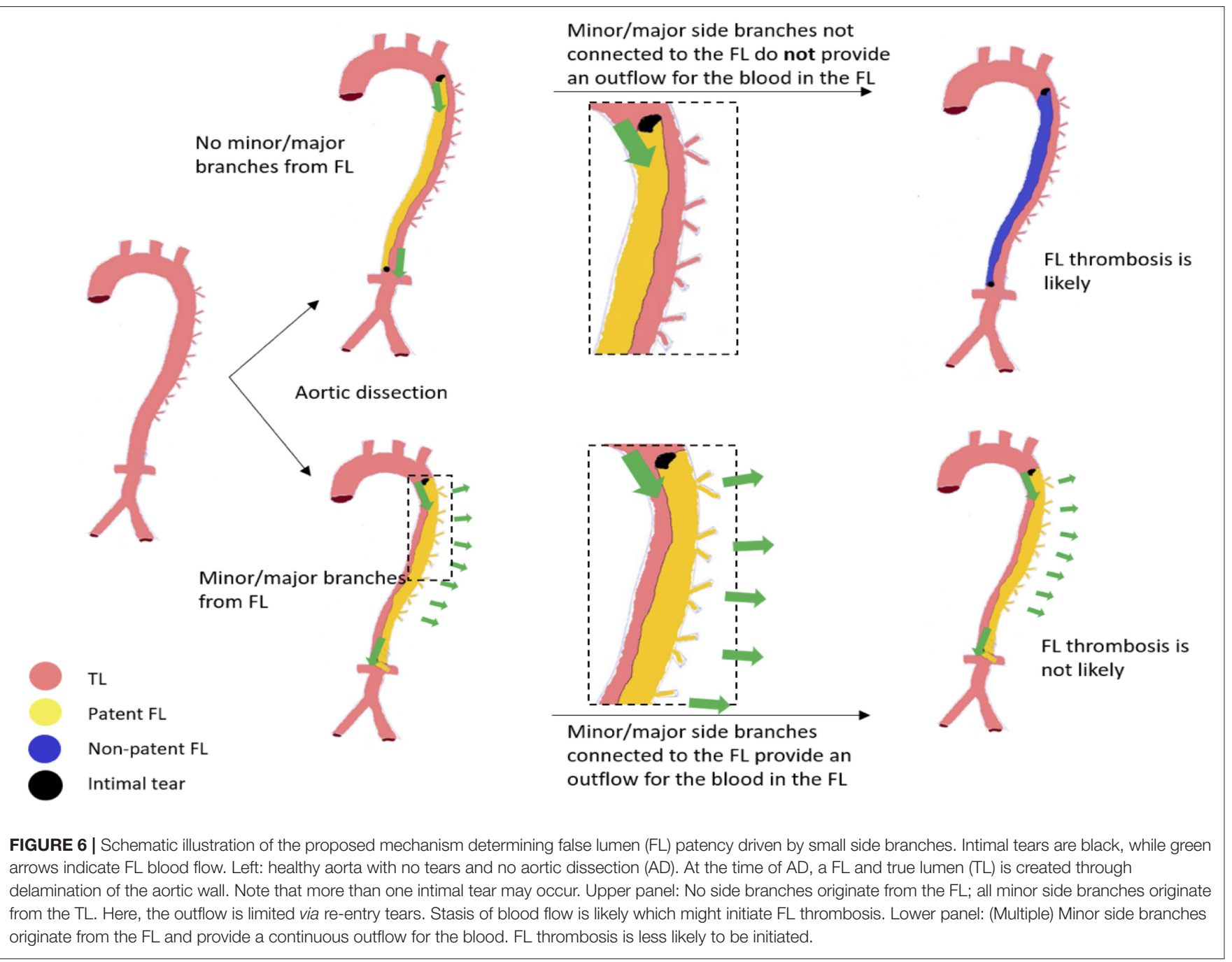

endovascular procedures needs thorough assessments of its feasibility in challenging anatomies. From a technical point of view, standardized imaging criteria may have to be set, and image processing tools (ideally based on artificial intelligence to favor automation) will have to be developed and validated for a timeefficient and objective quantification of (minor) side branches. Ideally, potential future clinical studies may be complemented with fundamental (bioengineering) research, to provide insights on how false lumen patency is (biomechanically) linked to patient outcome and disease progression. Here, realistic computational fluid-structure interaction models accounting for in- and outflow of the FL via intimal tears and side branches may help to elucidate the pressure built-up within the FL and mechanical stresses within the aortic wall. Of note, such models may also consider thrombosis $(42,43)$.

In conclusion, we have demonstrated that (minor) side branches that originate from the FL in TBAD patients are closely correlated with FL patency. We hypothesize that this is due to outflow from the FL into the branches. Our data advocate that controlling the outflow might be equally important to controlling the inflow in the treatment of patients with TBAD.

\section{DATA AVAILABILITY STATEMENT}

The raw data supporting the conclusions of this article will be made available by the authors, without undue reservation.

\section{ETHICS STATEMENT}

The studies involving human participants were reviewed and approved by Ethic committee of the Medical Faculty at the University Hospital Düsseldorf, Germany (ID: 2017064325) and the Ethic Committee at the Univeristy Hospital Ghent, Belgium (EC/2017/1635). Written informed consent for participation was not required for this study in accordance with the national legislation and the institutional requirements.

\section{AUTHOR CONTRIBUTIONS}

GL, BT, PS, HS, and MUW designed the study. JDB, JM, PD, FV, and IVH acquired the data. GL performed the computation. BT, PS, IVH, and MUW supervised the progress of the study. GL, BT, PS, JM, HS, and MUW analyzed and interpretated the data 
and drafted the manuscript. JDB, PD, FV, and IVH revised the manuscript for critical intellectual content. All authors approved the final version of the manuscript.

\section{FUNDING}

This work was supported by the Deutsche Forschungsgemeinschaft (DFG) [(SFB TRR259 (397484323) to JM, HS, and MUW], a local research grant from the Research Commission at the Medical Faculty of the Heinrich-HeinrichUniversity to MUW and by a research grant of the Fund for Scientific Research-Flanders (FWO G086917N) to PS and JDB.

\section{SUPPLEMENTARY MATERIAL}

The Supplementary Material for this article can be found online at: https://www.frontiersin.org/articles/10.3389/fcvm. 2021.710603/full\#supplementary-material

Supplementary Figure 1 | Overview of the number of Computed tomography (CT)-scans included in this study. Each dot represents a CT-scan. The $\mathrm{x}$-axis

\section{REFERENCES}

1. Riambau V, Böckler D, Brunkwall J, Cao P, Chiesa R, Coppi G, et al. Editor's choice - management of descending thoracic aorta diseases. Eur J Vasc Endovasc Surg. (2017) 53:4-52. doi: 10.1016/j.ejvs.2016.06.005

2. Wells CM, Subramaniam K. Acute aortic syndrome. Anesth Perioper Care Aortic Surg. (2011) 5:17-36. doi: 10.1007/978-0-387-85922-4_2

3. Clough RE, Taylor PR. A new classification system for aortic dissection. Eur J Vasc Endovasc Surg. (2013) 46:191. doi: 10.1016/j.ejvs.2013.05.009

4. Clough RE, Nienaber CA. Management of acute aortic syndrome. Nat Rev Cardiol. (2015) 12:103-14. doi: 10.1038/nrcardio.2014.203

5. Clough RE, Barillà D, Delsart P, Ledieu G, Spear R, Crichton S, et al. Editor's choice - long-term survival and risk analysis in 136 consecutive patients with type B aortic dissection presenting to a single centre over an 11 year period. Eur J Vasc Endovasc Surg. (2019) 57:633-8. doi: 10.1016/j.ejvs.2018.08.042

6. Acosta S, Blomstrand D, Gottsäter A. Epidemiology and long-term prognostic factors in acute type B aortic dissection. Ann Vasc Surg. (2007) 21:41522. doi: 10.1016/j.avsg.2007.01.012

7. Luebke T, Brunkwall J. Type B aortic dissection: a review of prognostic factors and meta-analysis of treatment options. AORTA. (2014) 2:26578. doi: 10.12945/j.aorta.2014.14-040

8. Nienaber CA, Rousseau H, Eggebrecht H. Randomized comparison of strategies for type B aortic dissection the investigation of STEnt grafts in aortic dissection (INSTEAD) Trial. Circulation. (2009) 120:251928. doi: 10.1161/CIRCULATIONAHA.109.886408

9. Fattori R, Montgomery D, Lovato L, Kische S, Di Eusanio M, Ince H, et al. Survival after endovascular therapy in patients with type B aortic dissection. JACC Cardiovasc Interv. (2013) 6:876-82. doi: 10.1016/j.jcin.2013.05.003

10. Ueki C, Sakaguchi G, Shimamoto T, Komiya T. Prognostic factors in patients with uncomplicated acute type B aortic dissection. Ann Thorac Surg. (2014) 97:767-73. doi: 10.1016/j.athoracsur.2013.10.038

11. Brunkwall J. Endovascular repair of acute uncomplicated aortic type B dissection promotes aortic remodelling : 1 year results of the ADSORB trial. Eur J Vasc Endovasc Surg. (2014) 48:285-91. doi: 10.1016/j.ejvs.2014.05.012

12. Hagan PG, Nienaber CA, Isselbacher EM, Bruckman D, Karavite DJ, Russman $\mathrm{PL}$, et al. The international registry of acute aortic dissection (IRAD). JAMA. (2000) 283:897. doi: 10.1001/jama.283.7.897

13. Tsai TT, Fattori R, Trimarchi S, Isselbacher E, Myrmel T, Evangelista A, et al. Long-term survival in patients presenting with type B acute aortic dissection: insights from the international registry of acute aortic dissection. Circulation. (2006) 114:2226-31. doi: 10.1161/CIRCULATIONAHA.106.622340 displays the time interval from the baseline CT-scan (upper panel) or from the time of thoracic endovascular repair (TEVAR) placement (lower panel) in months. A random value was used on the vertical axis to allow for the dots to be dispersed. At onset, all 89 patients were in the best medical treatment (BMT) group and received a baseline CT-scan. Of these, 37 patients underwent TEVAR during the further follow-up and were included in the sub-study. Here, the last CT-scan prior to TEVAR was considered as TEVAR baseline.

Supplementary Figure 2 | Distribution of the number and size of intimal tears, the distance from the first intimal tear to the left subclavian artery (LSA), the relative false lumen $(\mathrm{FL})$ area, and the number of major and minor side branches originating from the FL across the two specialized Vascular Surgery Departments. UKD: Department of Vascular and Endovascular Surgery at the University Hospital Düsseldorf, Germany; UZG: Department of Thoracic and Vascular Surgery at the University Hospital Ghent, Belgium.

Supplementary Figure $\mathbf{3}$ | Upper row: Correlation between the patent false lumen $(F L)$ length and the number of minor side branches originating from the FL at 3-, 24-, and 48-months follow-up for patients receiving thoracic endovascular repair (TEVAR). Spearman's rank correlation was applied, and the corresponding $p$-value is displayed. Lower row: Forest-plot of standardized beta values for a multiple linear regression model of the axial length of the patent FL for the same time points. At 48 months follow-up, the model matrix was rank deficient and stent graft coverage was not estimable (also see Figure $\mathbf{5}$ for 12 months follow-up).

14. Pape LA, Awais M, Wonznicki E, Suzuki T, Trimarchi S, Evangelista A, et al. Presentation, diagnosis, and outcomes of acute aortic dissection. J Am Coll Cardiol. (2015) 66:350-8. doi: 10.1016/j.jacc.2015.05.029

15. Song J-M, Kim S-D, Kim J-H, Kim M-J, Kang D-H, Seo JB, et al. Long-term predictors of descending aorta aneurysmal change in patients with aortic dissection. J Am Coll Cardiol. (2007) 50:799-804. doi: 10.1016/j.jacc.2007.03.064

16. Tsai TT, Evangelista A, Nienaber CA, Myrmel T, Meinhardt G, Cooper JV, et al. Partial thrombosis of the false lumen in patients with acute type B aortic dissection. N Engl J Med. (2007) 357:349-59. doi: 10.1056/NEJMoa063232

17. Evangelista A, Salas A, Ribera A, Ferreira-gonza I, Bijnens B. Longterm outcome of aortic dissection with patent false lumen predictive role of entry tear size and location. Circulation. (2012) 125:313341. doi: 10.1016/j.jvs.2012.09.028

18. Higashigaito K, Sailer AM, van Kuijk SMJ, Willemink MJ, Hahn LD, Hastie TJ, et al. Aortic growth and development of partial false lumen thrombosis are associated with late adverse events in type B aortic dissection. J Thorac Cardiovasc Surg. (2021) 161:1184-90.e2. doi: 10.1016/j.jtcvs.2019.10.074

19. Qin Y-L, Deng G, Li T-X, Jing R-W, Teng G-J. Risk factors of incomplete thrombosis in the false lumen after endovascular treatment of extensive acute type B aortic dissection. J Vasc Surg. (2012) 56:12328. doi: 10.1016/j.jvs.2012.04.019

20. Clough RE, Albayati MA, Donati T, Taylor PR. Uncomplicated type B dissections: which patients should be treated? Lessons learned from the recent literature. J Cardiovasc Surg (Torino). (2014) 55:145-50.

21. Sueyoshi E, Sakamoto I, Uetani M. Growth rate of affected aorta in patients with type B partially closed aortic dissection. Ann Thorac Surg. (2009) 88:1251-7. doi: 10.1016/j.athoracsur.2009.06.023

22. Hofferberth SC, Nixon IK, Mossop PJ. Aortic false lumen thrombosis induction by embolotherapy (AFTER) following endovascular repair of aortic dissection. J Endovasc Ther. (2012) 19:538-45. doi: 10.1583/JEVT-12-3844R.1

23. Hathcock JJ. Flow effects on coagulation and thrombosis. Arterioscler Thromb Vasc Biol. (2006) 26:1729-37. doi: 10.1161/01.ATV.0000229658.76797.30

24. Daugherty A, Cassis LA. Mouse models of abdominal aortic aneurysms. Arterioscler Thromb Vasc Biol. (2004) 24:42934. doi: 10.1161/01.ATV.0000118013.72016.ea

25. Lu G, Su G, Davis JP, Schaheen B, Downs E, Roy RJ, et al. A novel chronic advanced stage abdominal aortic aneurysm murine model. J Vasc Surg. (2016) 66:232-42.e4. doi: 10.1016/j.jvs.2016.07.105

26. Trachet B, Aslanidou L, Piersigilli A, Fraga-Silva RA, Sordet-Dessimoz J, Villanueva-Perez P, et al. Angiotensin II infusion into ApoE-/- mice: a model 
for aortic dissection rather than abdominal aortic aneurysm? Cardiovasc Res. (2017) 113:1230-42. doi: $10.1093 / \mathrm{cvr} / \mathrm{cv} \times 128$

27. Logghe G, Trachet B, Aslanidou L, Villaneuva-Perez P, De Backer J, Stergiopulos N, et al. Propagation-based phase-contrast synchrotron imaging of aortic dissection in mice: from individual elastic lamella to 3D analysis. Sci Rep. (2018) 8:2223. doi: 10.1038/s41598-018-20673-x

28. Tanaka A, Sakakibara M, Ishii H, Hayashida R, Jinno Y, Okumura $\mathrm{S}$, et al. Influence of the false lumen status on clinical outcomes in patients with acute type B aortic dissection. J Vasc Surg. (2014) 59:3216. doi: 10.1016/j.jvs.2013.08.031

29. Li D, Ye L, He Y, Cao X, Liu J, Zhong W, et al. False lumen status in patients with acute aortic dissection: a systematic review and meta-analysis. J Am Hear Assoc. (2016) 5:e003172. doi: 10.1161/JAHA.115.003172

30. Simring D, Raja J, Morgan-Rowe L, Hague J, Harris PL, Ivancev K. Placement of a branched stent graft into the false lumen of a chronic type B aortic dissection. J Vasc Surg. (2011) 54:1784-7. doi: 10.1016/j.jvs.2011.05.053

31. Verhoeven EL, Paraskevas KI, Oikonomou K, Yazar O, Ritter W, Pfister K, et al. Fenestrated and branched stent-grafts to treat post-dissection chronic aortic aneurysms after initial treatment in the acute setting. J Endovasc Ther. (2012) 19:343-9. doi: 10.1583/12-3860R.1

32. Kölbel T, Carpenter SW, Lohrenz C, Tsilimparis N, Larena-Avellaneda A, Debus ES. Addressing persistent false lumen flow in chronic aortic dissection: the knickerbocker technique. J Endovasc Ther. (2014) 21:11722. doi: 10.1583/13-4463MR-R.1

33. Rohlffs F, Tsilimparis N, Fiorucci B, Heidemann F, Debus ES, Kölbel T. The candy-plug technique: technical aspects and early results of a new endovascular method for false lumen occlusion in chronic aortic dissection. J Endovasc Ther. (2017) 24:549-55. doi: 10.1177/15266028177 09252

34. Zhang R, Zhou J, Feng J, Zhao Z, Liu J, Li Z, et al. Inducing false lumen thrombosis for retrograde type A aortic dissection. J Thorac Cardiovasc Surg. (2017) 153:57-65. doi: 10.1016/j.jtcvs.2016.09.022

35. Kamman AV, Brunkwall J, Verhoeven EL, Heijmen RH, Trimarchi S, Kasprzak P, et al. Predictors of aortic growth in uncomplicated type $\mathrm{B}$ aortic dissection from the Acute Dissection Stent Grafting or Best Medical Treatment (ADSORB) database. J Vasc Surg. (2017) 65:96471.e3. doi: 10.1016/j.jvs.2016.09.033

36. Magee GA, Yi JA, Kuwayama DP. Intercostal artery embolization to induce false lumen thrombosis in type B aortic dissection. J Vasc Surg Cases Innov Tech. (2020) 6:433-7. doi: 10.1016/j.jvscit.2020.05.012
37. Kotani S, Inoue Y, Kasai M, Suzuki S, Hachiya T. Modified 'candy-plug' technique for chronic type B aortic dissection with aneurysmal dilatation: a case report. J Cardiothorac Surg. (2017) 12:77. doi: 10.1186/s13019-017-0647-8

38. Bernard Y, Zimmermann H, Chocron S, Litzler J-F, Kastler B, Etievent J-P, et al. False lumen patency as a predictor of late outcome in aortic dissection. Am J Cardiol. (2001) 87:1378-82. doi: 10.1016/S0002-9149(01)01556-9

39. Akutsu K, Nejima J, Kiuchi K, Sasaki K. Effects of the patent false lumen on the long-term outcome of type B acute aortic dissection. Eur J Cardio-thoracic Surg. (2004) 26:359-66. doi: 10.1016/j.ejcts.2004.03.026

40. Tolenaar JL, van Keulen JW, Jonker FHW, van Herwaarden JA, Verhagen HJ, Moll FL, et al. Morphologic predictors of aortic dilatation in type B aortic dissection. J Vasc Surg. (2013) 58:1220-5. doi: 10.1016/j.jvs.2013.05.031

41. Trimarchi S, Tolenaar JL, Jonker FHW, Murray B, Tsai TT, Eagle KA, et al. Importance of false lumen thrombosis in type B aortic dissection prognosis. $J$ Thorac Cardiovasc Surg. (2013) 145:S208-12. doi: 10.1016/j.jtcvs.2012.11.048

42. Menichini $\mathrm{C}, \mathrm{Xu} \mathrm{XY}$. Mathematical modeling of thrombus formation in idealized models of aortic dissection: initial findings and potential applications. J Math Biol. (2016) 73:1-22. doi: 10.1007/s00285-016-0986-4

43. Menichini C, Cheng Z, Gibbs RGJ, Xu XY. Predicting false lumen thrombosis in patient-specific models of aortic dissection. $J$ R Soc Interface. (2016) 13:20160759. doi: 10.1098/rsif.2016.0759

Conflict of Interest: The authors declare that the research was conducted in the absence of any commercial or financial relationships that could be construed as a potential conflict of interest.

Publisher's Note: All claims expressed in this article are solely those of the authors and do not necessarily represent those of their affiliated organizations, or those of the publisher, the editors and the reviewers. Any product that may be evaluated in this article, or claim that may be made by its manufacturer, is not guaranteed or endorsed by the publisher.

Copyright $\odot 2021$ Logghe, Trachet, Segers, De Backer, Mulorz, Dueppers, Vermassen, Schelzig, Van Herzeele and Wagenhäuser. This is an open-access article distributed under the terms of the Creative Commons Attribution License (CC BY). The use, distribution or reproduction in other forums is permitted, provided the original author(s) and the copyright owner(s) are credited and that the original publication in this journal is cited, in accordance with accepted academic practice. No use, distribution or reproduction is permitted which does not comply with these terms. 